Research Article

\title{
Characteristics of Mesenchymal Stem Cells Are Independent of Bone Marrow Storage Temperatures
}

\author{
Valentin Schrodi, ${ }^{1,2}$ Claudia Neunaber $(\mathbb{D}),{ }^{1}$ Katrin Bundkirchen $\left(\mathbb{D}^{1},{ }^{1}\right.$ Weikang Ye, \\ Zhida Jiang, ${ }^{1}$ Maximilian Petri $\left(1,{ }^{1}\right.$ Christian Krettek $(1),{ }^{1}$ and Sandra Noack $(1)^{1}$ \\ ${ }^{1}$ Trauma Department, Hannover Medical School, 30625 Hannover, Germany \\ ${ }^{2}$ Department of Urology and Urooncology, Clinic of Braunschweig, 38126 Braunschweig, Germany \\ Correspondence should be addressed to Claudia Neunaber; neunaber.claudia@mh-hannover.de \\ Valentin Schrodi and Claudia Neunaber contributed equally to this work.
}

Received 18 May 2021; Revised 14 September 2021; Accepted 30 September 2021; Published 19 October 2021

Academic Editor: Yuriy Petrenko

Copyright (c) 2021 Valentin Schrodi et al. This is an open access article distributed under the Creative Commons Attribution License, which permits unrestricted use, distribution, and reproduction in any medium, provided the original work is properly cited.

\begin{abstract}
Mesenchymal stem cells play an important role in regenerative medicine due to their capability of self-renewal and multipotent differentiation. For research or clinical application, bone marrow aspirates are harvested during elective surgeries to isolate MSCs. If an immediate purification of the MSCs is not possible, the bone marrow must be stored. Therefore, the aim of this study was to investigate possible differences of stem cell characteristics regarding the self-renewal capability, the adipogenic, chondrogenic, and osteogenic differentiation, and the expression of surface antigens after different storage conditions of the bone marrow aspirates. Three groups were analysed: the first group was purified immediately after harvesting, the other two groups were processed after they were stored 18 to 24 hours at $22^{\circ} \mathrm{C}$ (room temperature) or at $4^{\circ} \mathrm{C}$. Comparisons between the groups were performed using the Kruskal-Wallis test for nonparametric data. The final results showed no significant difference between the different storage conditions. Therefore, storage of bone marrow aspirates for 18 to 24 hours at room temperature or $4^{\circ} \mathrm{C}$ is possible without loss of stem cell characteristics.
\end{abstract}

\section{Introduction}

Mesenchymal stem cells (MSCs) are multipotent self-renewable cells with the capability to differentiate into various cell types like adipocytes, chondrocytes, and osteoblasts [1]. They were primary isolated from bone marrow, but similar populations have also been detected in other human tissues like the dermis, adipose tissue, umbilical cord blood, and placenta [2-4].

Furthermore, MSCs are assumed to have immune modulatory functions as seen during osteoarthritis [5] by influencing the adaptive and innate immune system [6,7]. These several characteristics predestine MSCs to replace impaired cells or stimulate endogenous repair mechanisms [8]. The capability of mesenchymal stem cells to adhere and proliferate on cell culture surfaces allows them to expand in vitro [9]. Therefore, MSCs play a major role in regenerative medicine $[10,11]$. In the field of trauma and orthopaedic surgery, MSC-based concepts-for example, seeding of MSCs onto a scaffold [12] — are used as alternative methods to avoid possible complications of the current gold standard to treat large bone defects with autologous or allogeneous bone transplants $[13,14]$. However, MSCs are also used in many other clinical disciplines for instance by using the immunoregulatory effect to treat the graft-versus-host disease [15].

In order to develop further applications of stem cells for the clinical practice, research with MSCs is indispensable. In our department, the isolation of MSCs usually occurs immediately after the harvesting of bone marrow aspirates. At night or at weekends, a direct processing of the bone marrow is not possible due to staff shortages. In this case, the bone marrow aspirate has to be stored overnight. Therefore, the aim of our study was to investigate the influence of a longer 
TABLE 1: Fluorochrome-conjugated antibodies against CD antigens for flow cytometry analysis.

\begin{tabular}{lccccc}
\hline Antigen & Dye & Ig-class & Clone & Host & Company \\
\hline CD11b & APC & IgG1 k & ICRF44 & Mouse & Biolegend \\
CD13 & APC-Cy7 & IgG1 k & WM15 & Mouse & Biolegend \\
CD15 & FITC & IgG1 k & W6D3 & Mouse & Biolegend \\
CD29 & APC & IgG1 k & TS2/16 & Mouse & Biolegend \\
CD31 & FITC & IgG1 k & WM59 & Mouse & Biolegend \\
CD34 & PE-Cy7 & IgG1 k & 581 & Mouse & Biolegend \\
CD44 & FITC & IgG1 k & BJ18 & Mouse & Biolegend \\
CD45 & APC-Cy7 & IgG1 k & H130 & Mouse & Biolegend \\
CD54 & PE & IgG1 k & HCD54 & Mouse & Biolegend \\
CD56 & PE-Cy7 & IgG2a k & MEM-188 & Mouse & Biolegend \\
CD73 & APC & IgG1 k & AD2 & Mouse & Biolegend \\
CD90 & PerCP Cy5.5 & IgG1 k & 5E 07 & Mouse & Biolegend \\
CD105 & PE & IgG1 k & $43 A 3$ & Mouse & Biolegend \\
CD106 & APC & IgG1 k & STA & Mouse & Biolegend \\
CD146 & PE-Cy7 & IgG2a k & SHM-57 & Mouse & Biolegend \\
CD166 & FITC & IgG1 k & 3A6 & Mouse & MBL \\
CD271 & PerCP Cy5.5 & IgG1 k & ME20.4 & Mouse & Biolegend \\
CD274 & PE & IgG2b k & 29E.2A3 & Mouse & Biolegend \\
HLA-DR & PE & IgG2b k & LN3 & Mouse & Biolegend \\
Stro-1 & APC & IgM k & STRO-1 & Mouse & Biolegend \\
\hline
\end{tabular}

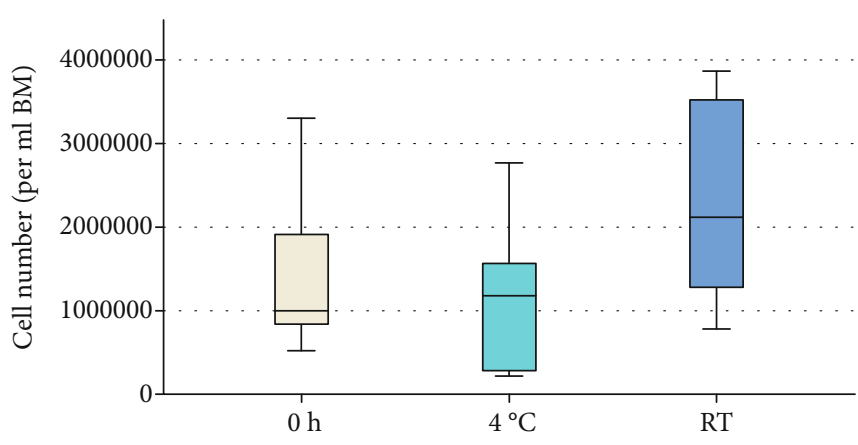

FIgURE 1: Cell number per ml BM in P0. The total number of cells per $\mathrm{ml} \mathrm{BM}$ in P0 was determined. There was no significant difference between the groups $(p=0.095 ; n=6)$.

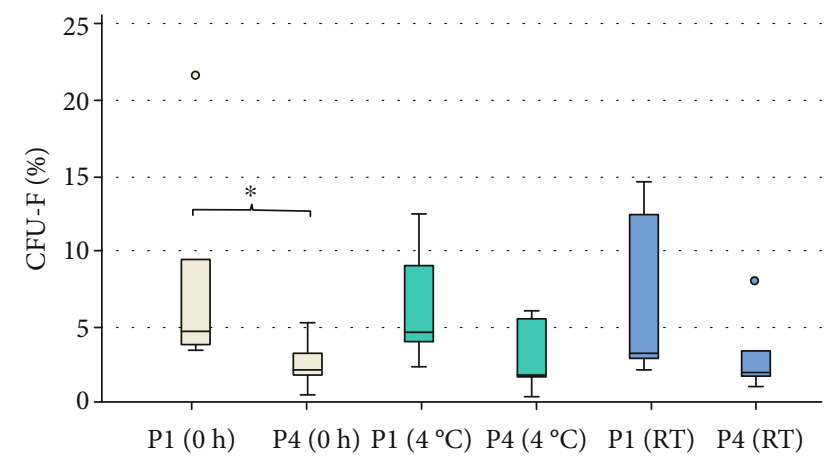

Figure 2: Percentage of CFU-F of the three experimental groups in $\mathrm{P} 1$ and P4. A significant difference was detected in group $0 \mathrm{~h}$ comparing passage 1 and $4\left({ }^{*} p=0.015 ; n=6\right)$. storage (18 to 24 hours) at different temperatures on the characteristics of human bone marrow-derived stem cells (hBMSCs) regarding the self-renewal capability, the adipogenic, chondrogenic, and osteogenic differentiation potential, and the expression of surface proteins.

\section{Materials and Methods}

2.1. Ethical Approval and Purification of hBMSCs. For studies involving human tissues, ethical approval of the study protocol and the process of purifying and analysing the hBMSCs was obtained by the ethical committee of the Hannover Medical School (Ethic No. 2562). Furthermore, a voluntary written informed consent was obtained from each donor, and all personal data were anonymised. A total of six donors were examined.

Human bone marrow was harvested by iliac crest aspiration during elective standard orthopaedic or trauma surgery at the Trauma Department of the Hannover Medical School. After immediate transfer of the bone marrow aspirate to the laboratory, the bone marrow was divided into three groups. The first group was purified immediately (group $0 \mathrm{~h}$ ), the other two groups were processed after they were stored 18 to 24 hours at room temperature $\left(\mathrm{RT} ; 22^{\circ} \mathrm{C}\right)$ or at $4^{\circ} \mathrm{C}$. The syringe in which the bone marrow was harvested was used for storage.

Afterwards, the hBMSCs were isolated by density gradient centrifugation for 30 minutes at $500 \times \mathrm{g}$ without brake using a synthetic polysaccharide-epichlorohydrin-copolymer (Biocoll ${ }^{\circledR}$, Biochrom, Berlin, Germany) as described before [16]. Shortly, the interphase which contains the mononuclear cells was extracted, washed, and seeded in MSC growth medium (DMEM FG 0415 (Biochrom)) with 10\% $(v / v)$ Fetal Bovine Serum (HyClone ${ }^{\circledR}$ FBS, Fisher Scientific, Schwerte, Germany), $20 \mathrm{mM}$ 4-(2-hydroxyethyl)-1-piperazineethanesulfonic acid (HEPES, Biochrom), $1 \%(100 \mathrm{U} / \mathrm{ml} / 100 \mu \mathrm{g} / \mathrm{ml})$ penicillin/streptomycin (Biochrom), and $2 \mathrm{ng} / \mathrm{ml}$ human recombinant fibroblast growth factor 2 (FGF2, PeproTech, Hamburg, Germany). The cells were now in passage $0(\mathrm{P} 0)$. Cells were cultured at $37^{\circ} \mathrm{C}$ and $5 \% \mathrm{CO}_{2}$ until a density of 70-80\% was reached [16]. Passaging was performed with $0.025 \%$ Trypsin-EDTA solution (Biochrom), and cells were seeded at a density of $2 \times 10^{3}$ cells per $\mathrm{cm}^{2}$ for the next passage. All experiments were performed in passage $4(\mathrm{P} 4)$ or as otherwise stated.

\subsection{Cell Amount and Colony Forming Unit-Fibroblast Assay} (CFU-F Assay). The total number of cells in $1 \mathrm{ml}$ of bone marrow was determined in P0 by counting the cells using a Neubauer counting chamber. To analyse the self-renewal potential of the hBMSCs, two CFU-assays were conducted in P1 and P4. Double determination of the cells was performed in six-well plates (Greiner Bio-One $\mathrm{GmbH}$, Frickenhausen, Germany) in decreasing concentrations of 500, 250, and 125 cells per well. CFUs were cultivated for 10 days at $37^{\circ} \mathrm{C}$ and $5 \% \mathrm{CO}_{2}$. Afterwards, cells were fixed with methanol (Merck, Darmstadt, Germany) and dyed for 30 minutes with $1 \%$ crystal violet solution (Merck). The colony number was counted to determine the percentage of clones per 100 seeded cells. 


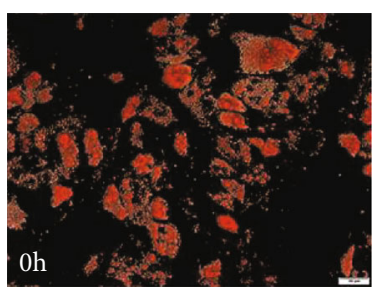

(a)

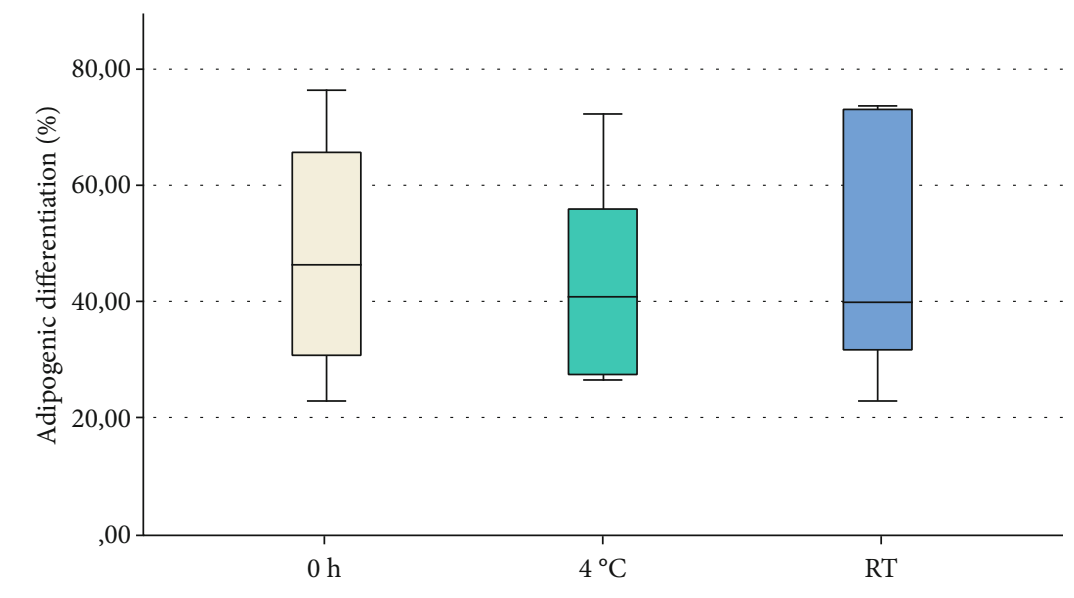

(d)

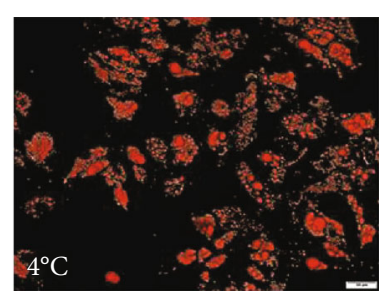

(b)

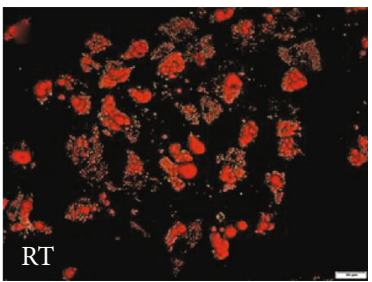

(c)

Figure 3: Adipogenic differentiation after 28 days. (a-c) Positive adipogenic differentiation was detected in all three groups ((a): 0 h; (b): $4^{\circ} \mathrm{C}$; and (c): RT) after staining with Oil Red O. The scale bar corresponds to $50 \mu \mathrm{m}$. (d) No significant difference regarding the adipogenic differentiation potential was detected $(p=0.796 ; n=6)$.

2.3. Flow Cytometry (FC). For flow cytometry $1 \times 10^{7}$ cells were used. In P4, cells were detached using $0.025 \%$ trypsin-EDTA solution and washed twice with FC buffer $(2 \%(v / v)$ FBS in PBS). The centrifugation steps were performed at $400 \times \mathrm{g}$ and $4^{\circ} \mathrm{C}$ for two minutes. Afterwards, the cells were incubated with appropriate fluorochromeconjugated antibodies (Biolegend, Koblenz, Germany; Table 1) for 60 minutes at $4^{\circ} \mathrm{C}$ in the dark. This process was followed by two washing steps with FC buffer. The cells were analysed on a FACS Canto (BD Biosciences, Heidelberg, Germany) recording $3 \times 10^{4}$ cells as described before [17]. Dead cells were excluded by using scatter parameters using the BD FACS Diva Software and Flowing Software version 2.5.0.

2.4. In Vitro Differentiation and Histological Staining. The adipogenic, chondrogenic, and osteogenic differentiation potential of stem cells was examined by inducing the process with convenient differentiation media. Each induced cell group had a concomitant control group. All stem cells were stored in a cell culture incubator at $37^{\circ} \mathrm{C}$ and $5 \% \mathrm{CO}_{2}$.

2.4.1. Adipogenic In Vitro Differentiation. For adipogenic differentiation, hBMSCs were seeded in six-well plates containing 150,000 cells per well. After 24 hours, the differentiation process was initiated by adipogenic medium consisting of DMEM FG0435 (500 ml ( $v / v)$, Biochrom), dexamethasone $(1 \mu \mathrm{M}(v / v)$, Sigma Aldrich), indomethacin $(60 \mu \mathrm{M}$ $(v / v)$, Sigma Aldrich), 3-isobutyl-1-methylxanthine (IBMX, $500 \mu \mathrm{M}(v / v)$, Sigma Aldrich), insulin $(10 \mu \mathrm{g} / \mathrm{ml}(v / v)$,
Sigma Aldrich), 4-(2-hydroxyethyl)-1-piperazineethanesulfonic acid (HEPES, $20 \mathrm{mM}(v / v)$, Biochrom), penicillin/streptomycin (1\% $(v / v)$, Biochrom), and Fetal Bovine Serum (FBS, 20\% $(v / v)$, Hyclone $\left.{ }^{\circledR}\right)$. The control medium consists of DMEM FG0415 $(500 \mathrm{ml}(v / v))$, 4-(2-hydroxyethyl)-1-piperazineethanesulfonic acid (HEPES, $20 \mathrm{mM}$ $(v / v))$, Fetal Bovine Serum (FBS, 10\% $(v / v)$, Hyclone $\left.{ }^{\circledR}\right)$, and penicillin/streptomycin $(1 \%(v / v))$ and was also used for the control group of the osteogenic differentiation. Media were replaced every 7 days. Cells for histological analysis were fixed in $4 \%$ formalin solution on days 0,7 , 14, 21, and 28 and stained with Oil Red O (5g/l in $60 \%$ Isopropanol, Roth) for 25 minutes. Photographs of each well with a 100x magnification were taken on the microscope CKX41 (Olympus, Tokyo, Japan). For quantitative analysis, the degree of adipogenic differentiation was assigned by evaluating the average of the stained area related to the total area. Therefore, a self-written image processing tool that referred to the OpenCV library (version 4.1.0) was used. Three representative images were assessed to ensure valid results. Based on different hue and saturation values, the stained area was kept apart from the background. The threshold levels were set manually by evaluating the specific parameters using a representative image. This data was used for the whole examination to facilitate a consistent assessment.

2.4.2. Osteogenic In Vitro Differentiation. Except the differentiation medium and the days of fixation, the osteogenic differentiation procedure was identical to the adipogenic differentiation 

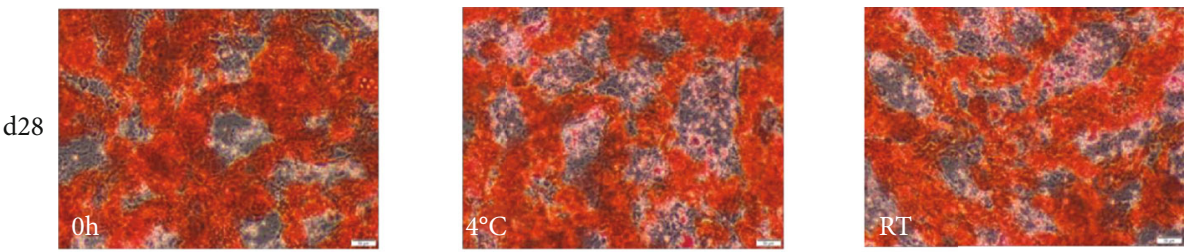

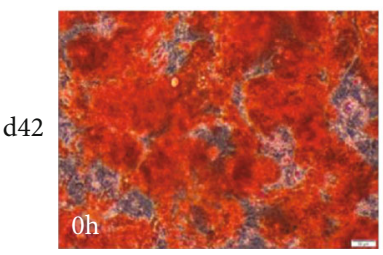

(a)

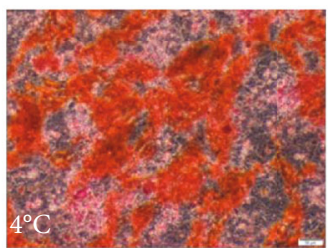

(b)

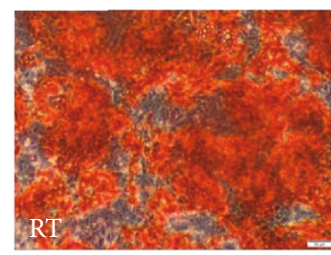

(c)

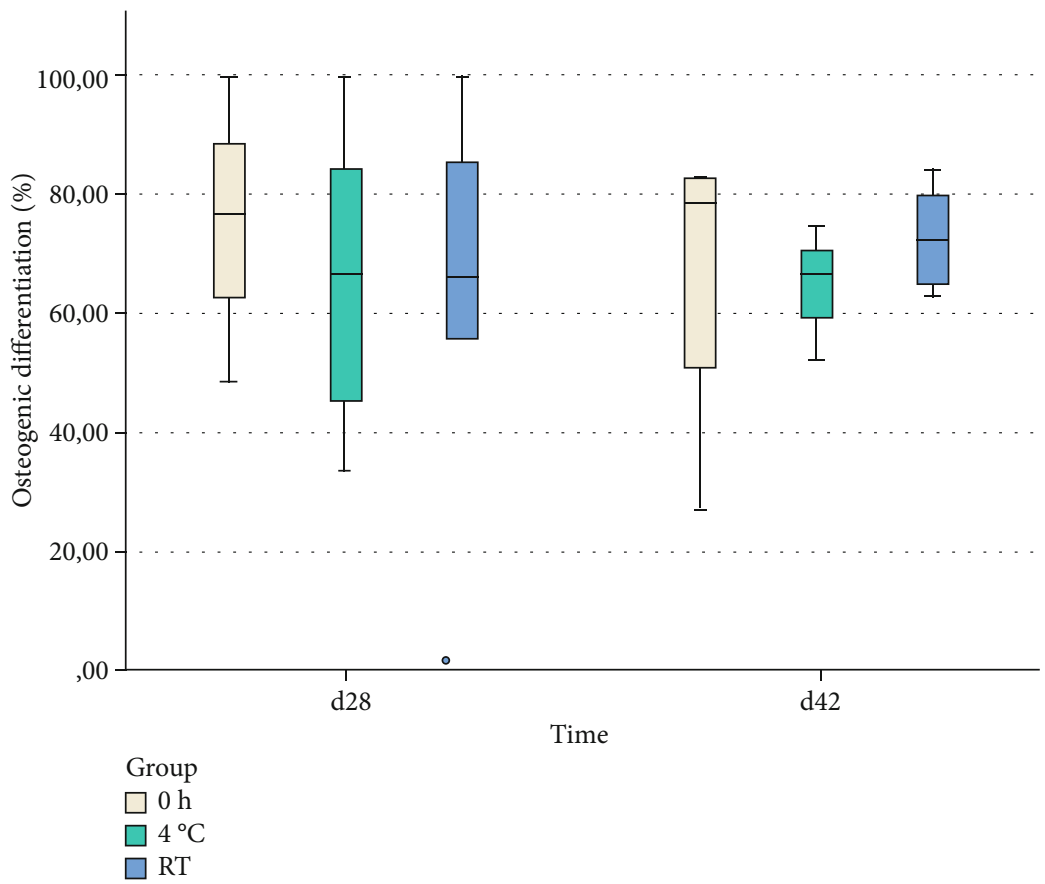

(d)

Figure 4: Osteogenic differentiation after 28 and 42 days after staining with Alizarin-Red S. (a-c) Osteogenesis was observed in all samples ((a): $0 \mathrm{~h}$; (b): $4^{\circ} \mathrm{C}$; and (c): RT) on days 28 (i) and 42 (ii). The scale bar corresponds to $50 \mu \mathrm{m}$. (d) No significant differences between the groups were found on both examination dates (day 28: $p=0.803$; day 42: $p=0.472 ; n=4$ ).

described above. The differentiation medium for osteogenesis consisted of DMEM FG0415 (500 ml $(v / v))$, 4-(2-hydroxyethyl)-1-piperazineethanesulfonic acid (HEPES, $20 \mathrm{mM}(v / v$ ) ), Fetal Bovine Serum (FBS, 10\% $(v / v)$, Hyclone $\left.{ }^{\circledR}\right)$, penicillin/streptomycin $(1 \%(v / v))$, dexamethasone $(100 \mathrm{nM}(v / v))$, ascorbate-2-phosphate $(500 \mathrm{ng} / \mathrm{ml}(v / v))$, and $\mathrm{Na}_{2} \mathrm{HPO}_{4} /$ $\mathrm{NaH}_{2} \mathrm{PO}_{4}(3 \mathrm{mM}(v / v), \mathrm{pH} 7,4$, Merck) as phosphate source. The histological fixation in $4 \%$ formalin solution occurred on days 28 and 42 . The samples were stained 10 minutes in the dark with 0.5\% Alizarin-Red S (Fluka, Germany) at pH4.5. The quantitative analysis was performed in the same way as described for the adipogenesis (section 2.4.1).

2.4.3. Chondrogenic In Vitro Differentiation. For chondrogenic differentiation, 250,000 hBMSCs were resuspended in control medium (DMEM FG0435 (500 ml ( $v / v)$, 4-(2-hydro- xyethyl)-1-piperazineethanesulfonic acid (HEPES, $20 \mathrm{mM}(v / v$ ) ), penicillin/streptomycin $(1 \%(v / v))$, dexamethasone $(0,1 \mu \mathrm{M}$ $(v / v)$ ), Insulin-Transferrin-Selenium (ITS, $10 \mu \mathrm{l} / \mathrm{ml}(v / v)$, Sigma Aldrich), ascorbate-2-phosphate (170 $\mu \mathrm{M}(v / v)$, Sigma Aldrich), sodium-pyruvate (1 mM ( $v / v)$, Biochrom), and proline $(350 \mu \mathrm{M}$ $(v / v)$, Roth) and centrifuged to generate the required pellet form. Afterwards, the pellets were cultivated in differentiation medium (control medium with additional transforming growth factor- $\beta 3$ (TGF- $\beta 3,10 \mathrm{ng} / \mathrm{ml} / 1.1 \mu \mathrm{l}(v / v)$, Peprotech)). Media were replaced every 7 days. For histological analysis, differentiation was stopped on days $7,14,21$, and 28 , and were pellets fixed in $4 \%$ formalin solution, embedded in Tissue Tek (OTC blue, Sakura Finetek, Staufen, Germany), and frozen in liquid nitrogen. Histological slices of $5 \mu \mathrm{m}$ were generated with a cryomicrotome (Microm HM 500 OM, Microm, Walldorf, Germany) and transferred onto coated microscope slides 


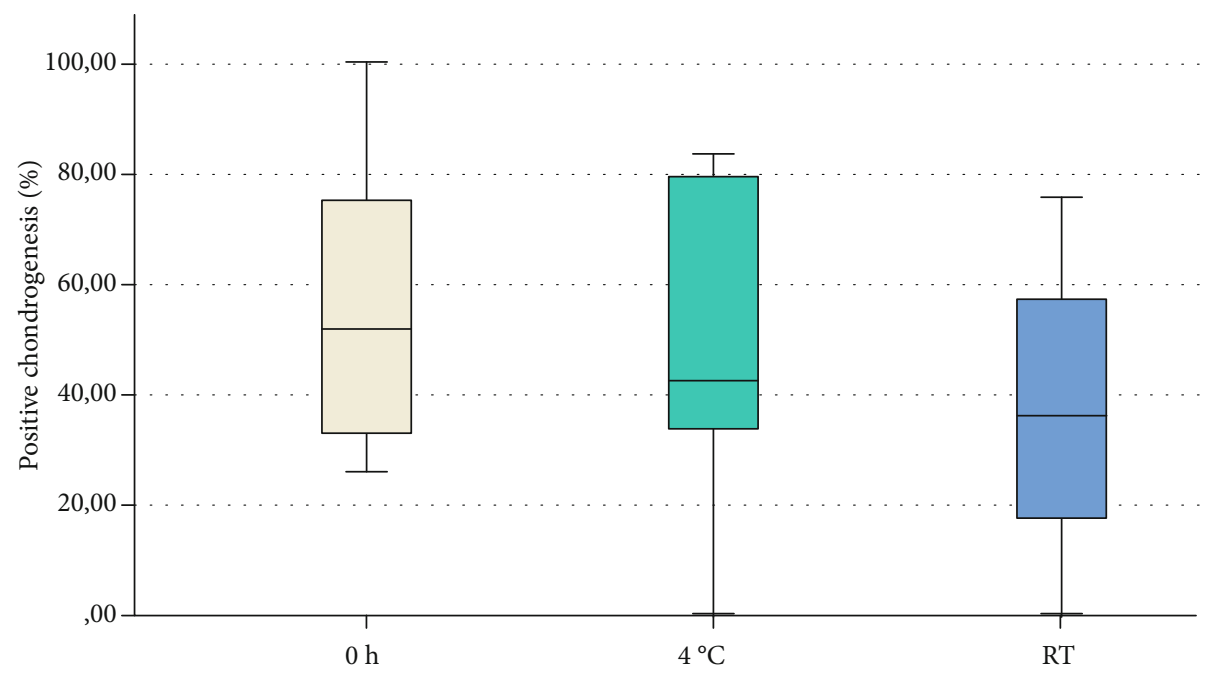

Figure 5: Positive chondrogenic differentiation after 28 days. The statistical examination revealed no significant difference regarding positive chondrogenesis $(p=0.590)$.

TABLE 2: Expression of specific surface proteins. Positive expression with a median higher than $90 \%$ was observed for the surface proteins CD13, CD29, CD44, CD73, CD90, CD105, and CD166 in all groups. The surface proteins CD11b, CD15, CD31, CD34, and CD45 were expressed less than $10 \%$ in median on stem cells.

\begin{tabular}{|c|c|c|c|c|c|c|c|c|c|}
\hline \multirow{2}{*}{ Surface protein } & \multicolumn{3}{|c|}{$0 \mathrm{~h}$} & \multicolumn{3}{|c|}{$4^{\circ} \mathrm{C}$} & \multicolumn{3}{|c|}{$\mathrm{RT}$} \\
\hline & Median & $Q_{1}$ & $Q_{3}$ & Median & $Q_{1}$ & $Q_{3}$ & Median & $Q_{1}$ & $Q_{3}$ \\
\hline CD13 & 97.4 & 95.0 & 98.0 & 98.1 & 97.0 & 98.4 & 98.0 & 97.1 & 98.4 \\
\hline CD29 & 98.6 & 97.3 & 100.0 & 98.5 & 97.0 & 99.0 & 98.7 & 98.0 & 99.0 \\
\hline CD44 & 98.6 & 97.8 & 100.0 & 98.2 & 97.9 & 98.8 & 98.3 & 97.3 & 98.3 \\
\hline CD73 & 98.8 & 98.3 & 99.6 & 98.5 & 98.2 & 99.6 & 98.7 & 98.3 & 98.8 \\
\hline CD90 & 95.9 & 94.7 & 99.0 & 96.7 & 88.1 & 97.6 & 95.3 & 87.1 & 97.6 \\
\hline CD105 & 99.3 & 98.4 & 100.0 & 98.6 & 98.4 & 99.1 & 98.8 & 98.7 & 99.0 \\
\hline CD166 & 98.5 & 98.0 & 99.0 & 98.7 & 98.6 & 99.0 & 98.6 & 98.6 & 98.6 \\
\hline CD11b & 1.1 & 0.0 & 1.6 & 1.0 & 1.0 & 2.0 & 1.4 & 0.4 & 2.0 \\
\hline CD15 & 0.4 & 0.2 & 1.0 & 0.7 & 0.2 & 1.4 & 0.4 & 0.0 & 1.0 \\
\hline CD31 & 1.8 & 1.2 & 2.6 & 3.1 & 1.4 & 4.6 & 2.7 & 1.6 & 5.7 \\
\hline $\mathrm{CD} 34$ & 0.8 & 0.0 & 5.0 & 1.0 & 0.0 & 7.0 & 1.1 & 0.0 & 7.0 \\
\hline CD45 & 1.1 & 0.4 & 3.0 & 1.5 & 0.5 & 1.8 & 1.1 & 0.9 & 2.0 \\
\hline
\end{tabular}

(SuperFrost Plus, Thermo Scientific, Darmstadt, Germany). After desiccation, samples were dyed with $0.1 \%$ safranin-O solution (Merck) for 15 minutes. After digitalization, the quantitative analysis was performed using two different groups to determine the successful progress of chondrogenesis. Chondrogenesis was graded as positive or negative differentiation. For evaluation, the different zones in the samples were marked. Afterwards, a grid was laid over the picture, and the boxes in the area of the two different groups were counted with a manual cell counter. As a result, the percentage of each group per chondrogenesis pellet was generated.

\section{Statistical Analyses}

Statistical analyses were performed using SPSS Statistics ${ }^{\circledR}$, version 24 (IBM SPSS Statistics Corp., New York, NY). All data were nonparametric. The primary outcome of the study was to detect significant differences between the three different storage conditions. Therefore, comparisons between the groups were performed using the Kruskal-Wallis test. Results are expressed as median and 95\% confidence interval (e.g., 5\% (CI: 3.90-9.53\%)). Graphical results are shown as boxplots or dot plots. A $p$ value of $\leq 0.05$ was considered significant.

\section{Results}

4.1. Cell Amount and CFU-F Assay. The total number of cells per $\mathrm{ml}$ bone marrow (BM) was calculated after immediate and delayed processing of the BM (Figure 1). No significant differences were detected between the groups $(p=0.095)$. 
The self-renewal capacity of hBMSCs was evaluated with CFU-F assays in P1 and P4 (Figure 2), and no significant differences were seen between the three different isolation groups. After immediate isolation of the BMSC $(0 \mathrm{~h}), 5 \%$ (CI: $3.90-9.53 \%)$ of all seeded cells formed a CFU. This is a significant difference compared to $\mathrm{P} 4$ of the same group in which only $2 \%$ (CI: $1.90-3.30 \%$ ) of the cells were able to form a colony $(p=0.015)$. No significant differences were detected between $\mathrm{P} 1$ and $\mathrm{P} 4$ for groups $4^{\circ} \mathrm{C}(\mathrm{P} 1$ : $5 \%$ (CI: 4.10-9.07\%) and P4: 2\% (CI: 1.77-5.67\%; $p=0.132)$ ) and RT (P1: 3\% (CI: 2.90-12.40\%) and P4: 2\% (CI: $1.77-3.50 \% ; p=0.177)$ ).

\subsection{In Vitro Differentiation of $h B M S C s$}

4.2.1. Adipogenic Differentiation. All stem cells which were cultured in the adipogenic differentiation medium exhibited an adipogenic differentiation (Figures 3(a)-3(c)). All control groups were negative. On day 28, the median of the three groups showed almost the same value with $46 \%$ (CI: $31-66 \%$ ) in group $0 \mathrm{~h} ; 41 \%$ (CI: $27-56 \%)$ in group $4^{\circ} \mathrm{C}$ and $40 \%$ (CI: $32-73 \%)$ in group RT ( $p=0.796$; Figure $3(d))$.

4.2.2. Osteogenic Differentiation. All stem cells which were cultured in the osteogenic differentiation medium exhibited an osteogenic differentiation on days 28 and 42 (Figures 4(a)4(c)). All control groups were negative. For both time points, no significant differences between the storage conditions (day 28: $p=0.803$ and day 42: $p=0.472$ ) were detected as shown in Figure 4(d).

4.2.3. Chondrogenic Differentiation. Chondrogenesis was observed in all induced groups except in two samples of different donors (one at room temperature and one at $4^{\circ} \mathrm{C}$ ). All control groups were negative. The examination of the positive chondrogenesis revealed no significant differences between the three groups ( $0 \mathrm{~h}: 51.62 \%(\mathrm{CI}: 33-75 \%) ; 4^{\circ} \mathrm{C}$ : 42.55\% (CI: 34-79\%); RT: 36.32\% (CI: $18-57 \%) ; p=0.590$; Figure 5).

4.3. Flow Cytometry (FC). The expression of 13 different surface proteins were analysed by flow cytometry. Regarding the expression of CD13, CD29, CD44, CD73, CD90, CD105, and CD166, the percentage median was over $90 \%$, and the surface proteins CD11b, CD15, CD31, CD34, and CD45 were expressed less than $10 \%$ in median on the stem cells (Table 2).

The Kruskal-Wallis test demonstrated no significant differences in expression of the reviewed positive (CD13: $p=0.241$; CD29: $p=0.821$; CD44: $p=0.566$; CD73: $p=0.873$; CD90: $p=0.738$; CD105: $p=0.587$; and CD166: $p=0.474)$ and negative (CD11b: $p=0.800$; CD15: $p=0.675$; CD31: $p=0.531$; CD34: $p=0.907$; and CD45: $p=0.920)$ surface proteins between the three experimental groups.

\section{Discussion}

The seeding of MSCs onto a scaffold to treat large bone defects is an important research area in orthopeadics, but in the clinical setting, it is not always possible to immediately isolate stem cells out of the bone marrow for research purposes.

Therefore, the aim of this study was to investigate the influence of an overnight storage of bone marrow at different temperatures on the characteristics of hBMSCs. To the best of our knowledge, there are no current publications that describe various storage conditions for bone marrow aspirates prior to isolation of the hBMSC. However, we have found some publication on the effects of various storage conditions after the isolation of the hBMSCs.

Sohn et al. investigated the influence of the duration of in vitro storage from MSCs on viability, self-renewal, and differentiation capability at $4^{\circ} \mathrm{C}$ and room temperature. With a longer time period, the differentiation potential was decreasing. The storage temperature had no significant influence regarding the cell quality [18]. This fact is congruent to our results where no significant difference was found comparing storage at $4^{\circ} \mathrm{C}$ and room temperature.

Among other parameters, another study compared the influence of different temperatures $\left(4^{\circ} \mathrm{C}, 37^{\circ} \mathrm{C}\right.$, and room temperature) on viability, differentiation capability, and expression of surface antigens of freshly and frozen-thawed MSCs. Storage of MSCs at room temperature or $37^{\circ} \mathrm{C}$ showed a lower viability than $4^{\circ} \mathrm{C}$. The analysis of surface antigens showed a similar expression pattern for fresh MSCs independent of the storage condition $\left(4^{\circ} \mathrm{C}\right.$ or room temperature) [19].

According to the minimal criteria for defining MSCs from the ISCT, the defining positive markers (CD73, CD90, and CD105) were also expressed on the MSCs in all groups in our attempt [20]. In addition to these positive markers, we examined surface proteins which should be negative on MSCs. The negative expression was congruent to results of other publications $[17,20,21]$. The expression of HLA-DR was not measured because the addition of FGF2 to MSC growth medium is known to induce the expression of this surface antigen [22].

To evaluate the self-renewal of the isolated hBMSCs, the CFU-F assay was used which is a scientifically established method to measure the clonogenic potential in a quantitative form $[23,24]$. The comparison of the three experimental groups within passages 1 and 4 revealed no statistical significant differences. Thus, we assumed that the examined storage conditions had no influence on the self-renewal of the stem cells. In passage 1, a higher number of colonies was observed than in passage 4 in all groups with statistical significance for group $0 \mathrm{~h}$. This circumstance is due to the replicative senescence of cells in culture which was also described by Schellenberg et al. In their experiment, the frequency of colony forming units declined with increasing number of cell passages [25].

At least some other previous studies examined the possibility of stem cell isolation from other tissue sources after storage. Perry et al. showed that the isolation of dental pulp-derived MSCs is possible for five days after tooth extraction and storing at $4^{\circ} \mathrm{C}$ and implied that an immediately preparation is not mandatory [26]. Adipose-derived stem cells can be used for long-term storage after isolation from adipose tissue. The sample should be stored at room temperature and used within 24 hours [27]. 


\section{Conclusion}

In summary, the results assumed no distinct favorite role of one examined storage condition for bone marrow aspirate. This indicates that a storage of bone marrow aspirates for 18 to 24 hours at room temperature or $4^{\circ} \mathrm{C}$ is possible without loss of stem cell characteristics concerning the adipogenic, chondrogenic, and osteogenic differentiation, as well as the self-renewal capacity and the expression of the evaluated surface proteins.

\section{Data Availability}

The datasets generated during and/or analysed during the current study are available from the corresponding author on reasonable request.

\section{Conflicts of Interest}

The authors declare that they have no conflicts of interest.

\section{Authors' Contributions}

Valentin Schrodi and Claudia Neunaber contributed equally to this work.

\section{Acknowledgments}

The study was financed by Else Kröner-Fresenius-Stiftung (grant 2016-A188). We would like to thank all participating patients for the bone marrow donation. Furthermore, we would like to thank Claudia Pütz and Melanie Weiss for their valuable assistance. The Institute of Functional and Applied Anatomy at Hannover Medical School generously made it possible for us to use their flow cytometer for this study. This work was part of the doctoral thesis of Valentin Schrodi.

\section{References}

[1] P. S. Frenette, S. Pinho, D. Lucas, and C. Scheiermann, "Mesenchymal stem cell: keystone of the hematopoietic stem cell niche and a stepping-stone for regenerative medicine," Annual Review of Immunology, vol. 31, no. 1, pp. 285-316, 2013.

[2] C. Vaculik, C. Schuster, W. Bauer et al., "Human dermis harbors distinct mesenchymal stromal cell subsets," The Journal of Investigative Dermatology, vol. 132, no. 3, 3 Part 1, pp. 563-574, 2012.

[3] W. Wagner, F. Wein, A. Seckinger et al., "Comparative characteristics of mesenchymal stem cells from human bone marrow, adipose tissue, and umbilical cord blood," Experimental Hematology, vol. 33, no. 11, pp. 1402-1416, 2005.

[4] J. R. Park, E. Kim, J. Yang et al., "Isolation of human dermis derived mesenchymal stem cells using explants culture method: expansion and phenotypical characterization," Cell and Tissue Banking, vol. 16, no. 2, pp. 209-218, 2015.

[5] A. I. Caplan, "Mesenchymal stem cells: the past, the present, the future," Cartilage, vol. 1, no. 1, pp. 6-9, 2010.

[6] M. E. Bernardo and W. E. Fibbe, "Mesenchymal stromal cells: sensors and switchers of inflammation," Cell Stem Cell, vol. 13, no. 4, pp. 392-402, 2013.
[7] A. Keating, "Mesenchymal stromal cells: new directions," Cell Stem Cell, vol. 10, no. 6, pp. 709-716, 2012.

[8] Z. Poon, W. C. Lee, G. Guan et al., "Bone marrow regeneration promoted by biophysically sorted osteoprogenitors from mesenchymal stromal cells," Stem Cells Translational Medicine, vol. 4, no. 1, pp. 56-65, 2015.

[9] D. C. Colter, R. Class, C. M. DiGirolamo, and D. J. Prockop, "Rapid expansion of recycling stem cells in cultures of plastic-adherent cells from human bone marrow," Proceedings of the National Academy of Sciences of the United States of America, vol. 97, no. 7, pp. 3213-3218, 2000.

[10] L. T. Wang, C. H. Ting, M. L. Yen et al., "Human mesenchymal stem cells (MSCs) for treatment towards immune- and inflammation-mediated diseases: review of current clinical trials," Journal of Biomedical Science, vol. 23, no. 1, p. 76, 2016.

[11] Y. Miura, "Human bone marrow mesenchymal stromal/stem cells: current clinical applications and potential for hematology," International Journal of Hematology, vol. 103, no. 2, pp. 122-128, 2016.

[12] L. Watson, S. J. Elliman, and C. M. Coleman, "From isolation to implantation: a concise review of mesenchymal stem cell therapy in bone fracture repair," Stem Cell Research \& Ther$a p y$, vol. 5, no. 2, p. 51, 2014.

[13] J. Stanovici, L. R. le Nail, M. A. Brennan et al., "Bone regeneration strategies with bone marrow stromal cells in orthopaedic surgery," Current Research in Translational Medicine, vol. 64, no. 2, pp. 83-90, 2016.

[14] G. Arealis and V. S. Nikolaou, "Bone printing: new frontiers in the treatment of bone defects," Injury, vol. 46, Supplement 8, pp. S20-S22, 2015.

[15] A. Uccelli, L. Moretta, and V. Pistoia, "Mesenchymal stem cells in health and disease," Nature Reviews Immunology, vol. 8, no. 9, pp. 726-736, 2008.

[16] L. M. Schäck, M. Buettner, A. Wirth et al., "Expression of CD24 in Human Bone Marrow-Derived Mesenchymal Stromal Cells Is Regulated by TGF $\beta 3$ and Induces a Myofibroblast-Like Genotype," Stem Cells International, vol. 2016, Article ID 1319578, 13 pages, 2016.

[17] L. M. Schäck, S. Noack, R. Weist et al., "Analysis of surface protein expression in human bone marrow stromal cells: new aspects of culture-induced changes, inter-donor differences and intracellular expression," Stem Cells and Development, vol. 22, no. 24, pp. 3226-3235, 2013.

[18] H. S. Sohn, J. S. Heo, H. S. Kim, Y. Choi, and H. O. Kim, "Duration of in vitro storage affects the key stem cell features of human bone marrow-derived mesenchymal stromal cells for clinical transplantation," Cytotherapy, vol. 15, no. 4, pp. 460-466, 2013.

[19] R. Pal, M. Hanwate, and S. M. Totey, "Effect of holding time, temperature and different parenteral solutions on viability and functionality of adult bone marrow-derived mesenchymal stem cells before transplantation," Journal of Tissue Engineering and Regenerative Medicine, vol. 2, no. 7, pp. 436-444, 2008.

[20] M. Dominici, K. le Blanc, I. Mueller et al., "Minimal criteria for defining multipotent mesenchymal stromal cells. The International Society for Cellular Therapy position statement," Cytotherapy, vol. 8, no. 4, pp. 315-317, 2006.

[21] M. T. Rojewski, B. M. Weber, and H. Schrezenmeier, "Phenotypic characterization of mesenchymal stem cells from various tissues," Transfusion Medicine and Hemotherapy, vol. 35, no. 3, pp. 168-184, 2008. 
[22] C. Bocelli-Tyndall, P. Zajac, N. di Maggio et al., "Fibroblast growth factor 2 and platelet-derived growth factor, but not platelet lysate, induce proliferation-dependent, functional class II major histocompatibility complex antigen in human mesenchymal stem cells," Arthritis and Rheumatism, vol. 62, no. 12, pp. 3815-3825, 2010.

[23] A. Harichandan and H. J. Bühring, "Prospective isolation of human MSC," Best Practice \& Research Clinical Haematology, vol. 24, no. 1, pp. 25-36, 2011.

[24] D. Gothard, J. I. Dawson, and R. O. C. Oreffo, "Assessing the potential of colony morphology for dissecting the CFU-F population from human bone marrow stromal cells," Cell and Tissue Research, vol. 352, no. 2, pp. 237-247, 2013.

[25] A. Schellenberg, Q. Lin, H. Schüler et al., "Replicative senescence of mesenchymal stem cells causes DNA-methylation changes which correlate with repressive histone marks," Aging (Albany NY), vol. 3, no. 9, pp. 873-888, 2011.

[26] B. C. Perry, D. Zhou, X. Wu et al., "Collection, cryopreservation, and characterization of human dental pulp-derived mesenchymal stem cells for banking and clinical use," Tissue Engineering. Part C, Methods, vol. 14, no. 2, pp. 149-156, 2008.

[27] B. Bunnell, M. Flaat, C. Gagliardi, B. Patel, and C. Ripoll, "Adipose-derived stem cells: isolation, expansion and differentiation," Methods, vol. 45, no. 2, pp. 115-120, 2008. 